\title{
Assistência Farmacêutica na atenção primária à saúde de um Municipio Potiguar, Brasil
}

\author{
Pharmaceutical Assistance at primary health care in \\ a Potiguar County, Brazil
}

Recebido em: $11 / 11 / 2018$ Aceito em: 23/04/2019

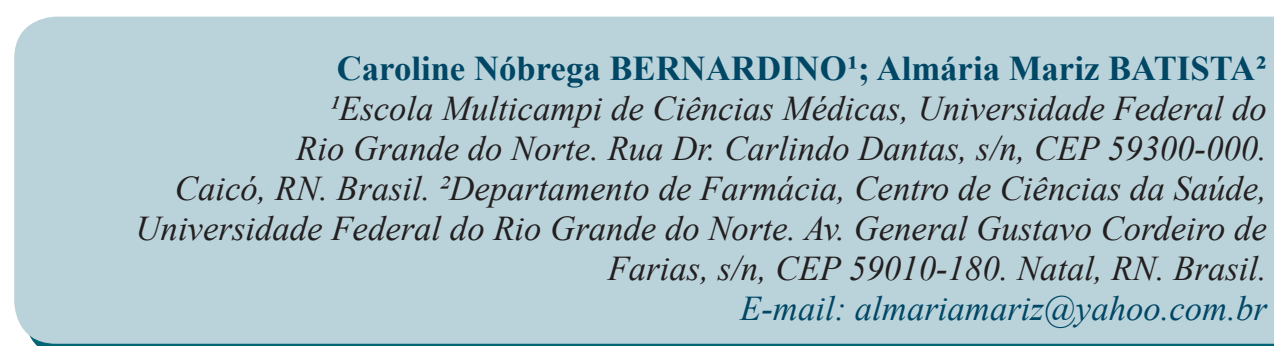

Caroline Nóbrega BERNARDINO ${ }^{1}$; Almária Mariz BATISTA ${ }^{2}$ ${ }^{1}$ Escola Multicampi de Ciências Médicas, Universidade Federal do
Rio Grande do Norte. Rua Dr. Carlindo Dantas, s/n, CEP 59300-000. Caicó, RN. Brasil. ${ }^{2}$ Departamento de Farmácia, Centro de Ciências da Saúde, Farias, s/n, CEP 59010-180. Natal, RN. Brasil. E-mail:almariamariz@yahoo.com.br

\section{ABSTRACT}

This study aimed to evaluate Pharmaceutical Care in Caicó, RN State, Brazil, through a transversal and descriptive study carried on from September 2016 to March 2017. An instrument with indicators was applied to the workers from the Pharmaceutical Supply Center and pharmacies of Health Basic Units. The city has its List of Essential Drugs, although there is not a Commission of Therapeutic and Pharmacy. No evaluated pharmacy presented a Pharmacist, Standard Operational Procedure, nor supply-handling. Register. The medicines dispensing was carried out by Community health agents (35\%), nurses (74\%) and nursing assistants (100\%), instead of Pharmacist. Air conditioners was observed at only $65 \%$ of the pharmacies, while no pharmacy had humidity and temperature control. Data reveal that Pharmaceutical Care in Caicó County was not efficient, and a commitment by municipal managers is necessary to provide an effective service to the Public Health Care users.

Keywords: Pharmaceutical Assistence; pharmaceutical services; primary health care

\section{RESUMO}

Este estudo teve como objetivo avaliar a Assistência Farmacêutica no município de Caicó, RN, Brasil, com base em indicadores, via estudo transversal e descritivo realizado de setembro de 2016 a março de 2017, a partir de 10 indicadores aplicados à Central de Abastecimento Farmacêutico e 30 aplicados nas 23 farmácias das Unidades Básicas de Saúde. O município possui Relação Municipal de Medicamentos Essenciais, mas não conta com Comissão de Farmácia e Terapêutica. Nenhuma das farmácias apresentava farmacêutico lotado, procedimento operacional padrão e registros de movimentação de estoque. A entrega de medicamentos nestas farmácias era realizada por agentes comunitários de saúde (35\%), enfermeiros (74\%) e técnicos de enfermagem (100\%). Apenas $65 \%$ das farmácias possuía ar condicionado e nenhuma delas apresentava mapa de controle de umidade e temperatura. Os dados revelam que a assistência farmacêutica do município de Caicó é ainda incipiente e pressupõe maior responsabilidade e compromisso dos gestores municipais para cumprir com sua efetividade.

Palavras-chave: Assistência Farmacêutica; indicadores; atenção primária à saúde 


\section{INTRODUÇÃO}

A Política Nacional de Assistência Farmacêutica define assistência farmacêutica como conjunto de ações voltadas a promoção, proteção e recuperação da saúde, tanto individual como coletiva, tendo o medicamento como insumo essencial e visando seu acesso e uso racional. Envolve pesquisa, desenvolvimento e produção de medicamentos e insumos, bem como sua seleção, programação, aquisição, distribuição, dispensação, garantia de qualidade de produtos e serviços, acompanhamento e avaliação de sua utilização, na perspectiva de resultados concretos e melhoria da qualidade de vida da população (1).

Sob a égide da Constituição Federal de 1988, a saúde passou a ser direito social e dever do Estado e a institucionalização do Sistema Único de Saúde (SUS), via Lei $n^{\circ} 8.080 / 90$, incluiu, neste âmbito, a assistência terapêutica integral, inclusive farmacêutica (2). Sob esta óptica, foi regulamentada a Política Nacional de Medicamentos, a fim de nortear, nas 3 esferas de governo, a promoção de acesso a medicamentos essenciais bem como seu uso racional (3). Outrossim, a Política Nacional de Atenção Básica apresenta como responsabilidade às 3 esferas de governo ações de assistência farmacêutica, garantindo acesso a medicamentos em conformidade com a Relação Nacional de Medicamentos Essenciais (RENAME) e a relação específica municipal e estadual nos pontos de atenção (4).

A institucionalização da assistência farmacêutica nos sistemas municipais de saúde ainda é parcial, sendo constatado, nas regiões do Brasil, um baixo percentual de existência de Comissão de Farmácia e Terapêutica (CFT) $(13,2 \%)$ e escassez nos investimentos em qualificação profissional (11,9\%), sobretudo do farmacêutico, necessário para estruturação dos processos da assistência farmacêutica (5).

Considerando que, no Brasil, há uma diversidade de municípios com diferentes estágios de organização e estruturação dos serviços de saúde, e contando com equipe de profissionais de composição diversa, é de se esperar que haja, também, diversidade na forma de desenvolvi- mento das atividades da assistência farmacêutica (6).

Dessa forma, o objetivo do estudo foi avaliar a Assistência Farmacêutica do município de Caicó, RN, por meio de um diagnóstico situacional, a partir da análise de indicadores selecionados.

\section{MÉTODO}

Trata-se de estudo transversal, descritivo, de caráter exploratório, desenvolvido no município de Caicó, RN, no período de setembro de 2016 a março de 2017.

Caicó é um município brasileiro pertencente ao Estado do Rio Grande do Norte (RN) com população estimada em 68.222 habitantes (7). Segundo a Secretaria Municipal de Saúde, o município apresenta 23 unidades básicas de saúde (UBS), sendo 18 na zona urbana e 5 na rural, além de 25 Equipes Saúde da Família (ESF) e 1 Núcleo de Apoio à Saúde da Família (NASF), categoria 1.

O objeto de pesquisa foi a Assistência Farmacêutica municipal, sendo os locais de estudo a Central de Abastecimento Farmacêutico (CAF) e as farmácias das 23 UBS deste município.

A coleta de dados foi realizada por meio da aplicação de indicadores selecionados a partir de metodologia proposta pela Organização Mundial de Saúde (OMS) para a avaliação de políticas farmacêuticas. A condução da pesquisa no Brasil foi atribuída à Organização Pan-Americana de Saúde / Ministério da Saúde (OPAS/MS) (8). Os indicadores foram divididos em 2 partes, sendo a primeira com 10 indicadores aplicados à $\mathrm{CAF}$, e a segunda, com 30 aplicados às farmácias das UBS.

Donabedian (1984) considerou três enfoques básicos de caracterização de indicadores de avaliação da qualidade: estrutura, processo e resultado (9). Indicadores de estrutura relacionam-se a características que influenciam na qualidade da atenção à saúde, como recursos financeiros, materiais e humanos. Indicadores de processo remetem à rentabilidade das atividades desempenhadas que compõem a atenção à saúde e à relação entre profissionais e população. Indicadores de resultado 
incluem modificações no estado de saúde da população, relacionadas com fatores de risco, avaliando efeitos a longo prazo, por isso, não foram aplicados ao estudo.

Os dados foram analisados por meio do programa Microsoft Excel ${ }^{\circledR}$ 2013. Os dados foram sistematizados e interpretados por análise descritiva mediante frequência absoluta/relativa.

Por não se tratar de pesquisa envolvendo seres humanos, não foi necessária aprovação por Comitê de Ética e Pesquisa; então, o estudo contou com assinatura de Termo de Fiel Depositário pela secretária de saúde do município, autorizando o acesso livre às UBS e à CAF para coleta de dados.

\section{RESULTADOS E DISCUSSÃO}

Foram destacadas, como fragilidades na assistência farmacêutica municipal, a inexistência de Comissão de Farmácia e Terapêutica e Serviço de Informações sobre Medicamentos, bem como ausência de diretrizes para doação de medicamentos e recolhimento de medicamentos vencidos/avariados. Quanto às condições das farmácias das UBS, em $100 \%$ não havia farmacêutico lotado, procedimentos operacionais padrão (POP), registro de movimentação de estoque, computador, impressora, acesso à internet, refrigerador, termômetro, fontes de informação sobre medicamentos e extintor de incêndio (Quadro 1 e Tabela 1).

Quadro 1. Indicadores para avaliação da assistência farmacêutica aplicados à Central de Abastecimento Farmacêutico (CAF) do município de Caicó, RN, Brasil.

\begin{tabular}{|c|c|c|}
\hline INDICADOR & DESCRIÇÃO & RESPOSTA \\
\hline \multirow{9}{*}{ ESTRUTURA } & Existe plano municipal de assistência farmacêutica? & Sim \\
\hline & Existe coordenação de assistência farmacêutica municipal? & Sim \\
\hline & Existe comissão de farmácia e terapêutica (CFT)? & Não \\
\hline & Existe relação municipal de medicamentos essenc, em ais (REMUME) (ou lista pactuada)? & Sim \\
\hline & Existe formulário terapêutico municipal? & Não \\
\hline & $\begin{array}{l}\text { Existe catálogo com especificações técnicas dos medicamentos para editais de aquisição } \\
\text { municipal? }\end{array}$ & Não \\
\hline & Existem diretrizes para doação de medicamentos? & Não \\
\hline & Existe plano de recolhimento de medicamentos vencidos/avariados? & Não \\
\hline & Existe serviço/centro de informação de medicamentos (SIM/CIM) acessível na cidade? & Não \\
\hline PROCESSO & $\begin{array}{l}\text { Há programação das necessidades de medicamentos de acordo com métodos } \\
\text { recomendados? }\end{array}$ & Sim \\
\hline
\end{tabular}

Caicó dispõe de Relação Municipal de Medicamentos Essenciais (REMUME), elaborada por farmacêuticos da CAF em reunião com outros profissionais de saúde a partir do perfil epidemiológico do município, uma vez que inexiste CFT. A versão mais recente é de 2014.

O gerenciamento da assistência farmacêutica deve contemplar a estruturação e a organização dos serviços, de modo a implementar todas as atividades pertinentes. Mesmo municípios de pequeno porte, com estrutura reduzida e serviços de menor complexidade, necessitam de organização e de gerenciamento eficientes (6). O desconhecimento, por parte da gestão municipal da saúde, dos benefí- cios e atribuições de uma CFT, bem como questões de disponibilidade e interesse de profissionais para compor a comissão talvez seja um dos problemas enfrentados pelo município de Caicó.

Um estudo de caracterização da assistência farmacêutica na atenção básica no Brasil constatou uma não consonância entre a existência de Listas de Medicamentos Essenciais (LME) (85,9\% Brasil/92\% Nordeste) e CFT (13,2\% Brasil/10,2\% Nordeste) (5). A existência de ambos são indicadores estruturais importantes no processo de seleção, portanto, a ausência de ao menos um destes não atende às expectativas da Política Nacional de Medicamentos (3) e compromete o processo de seleção. 
Em um estudo de avaliação de indicadores de uso racional de medicamentos em nível de atenção primária no cenário brasileiro, dispensadores relataram a disponibilidade de RENAME ou REMUME na UBS (89,5\% Brasi1/87,5\% Nordeste) (10), o que diverge do presente estudo, no qual apenas $48 \%$ das UBS a tinham disponível. A elaboração, por si só, de LME não garante a adesão à mesma por parte dos prescritores. É necessária, também, a sensibilização destes, o que pode ser feita por sua inclusão no processo de construção da LME, bem como pela disponibilidade do documento no local de trabalho.

Tabela 1. Indicadores de avaliação da assistência farmacêutica aplicados nas farmácias públicas do município de Caicó, RN, Brasil.

\begin{tabular}{|c|c|c|c|}
\hline INDICADOR & DESCRIÇÃO & $\operatorname{SIM} n(\%)$ & NÃO $n(\%)$ \\
\hline \multirow{22}{*}{ ESTRUTURA } & Há farmacêutico lotado? & & $23(100)$ \\
\hline & Há outro recurso humano na farmácia? & & $23(100)$ \\
\hline & Há local que permita contato direto funcionário-usuário? & $7(30)$ & $16(70)$ \\
\hline & Há Procedimento Operacional Padrão (POP)? & & $23(100)$ \\
\hline & Há registro de movimentação de estoque? & & $23(100)$ \\
\hline & Há medicamentos com data de validade expirada nas prateleiras? & $4(17)$ & $19(83)$ \\
\hline & Medicamentos são armazenados sem contato direto com solo e parede? & $13(56)$ & $10(44)$ \\
\hline & $\begin{array}{l}\text { Instalações com boas condições higiênico-sanitárias e de conservação (piso, } \\
\text { parede cor clara, fácil higienização)? }\end{array}$ & $13(56)$ & $10(44)$ \\
\hline & Farmácia limpa, sem poeira ou sujeira aparente? & $16(70)$ & $7(30)$ \\
\hline & Há evidências de insetos ou roedores no local? & $2(8)$ & $21(92)$ \\
\hline & Há REMUME ou lista pactuada disponível nas UBS? & $11(48)$ & $12(52)$ \\
\hline & Há área para fracionamento? & & $23(100)$ \\
\hline & Há ar condicionado? & $15(65)$ & $8(35)$ \\
\hline & Há refrigerador? & & $23(100)$ \\
\hline & Há termômetro? & & $23(100)$ \\
\hline & Há mapa para controle de temperatura? & & $23(100)$ \\
\hline & Há fontes de informação sobre medicamentos? & & $23(100)$ \\
\hline & Há computador? & & $23(100)$ \\
\hline & Há acesso à internet? & & $23(100)$ \\
\hline & Há impressora? & & $23(100)$ \\
\hline & Há lixeira? & $17(74)$ & $6(26)$ \\
\hline & Há extintor de incêndio? & & $23(100)$ \\
\hline \multirow{8}{*}{ PROCESSO } & Há treinamento continuado para funcionários da farmácia? & & $23(100)$ \\
\hline & Cartelas são cortadas para dispensação de quantidade específica? & $16(69)$ & $7(31)$ \\
\hline & Produtos estão protegidos da ação direta da luz solar? & $18(79)$ & $5(21)$ \\
\hline & A área é livre de umidade? & $17(74)$ & $6(26)$ \\
\hline & Prescrições são arquivadas na farmácia após dispensação? & $22(96)$ & $1(4)$ \\
\hline & Produtos recebidos são conferidos quanto a quantidade, validade e qualidade? & $23(100)$ & \\
\hline & Medicamentos são armazenados de forma sistemática? & $17(74)$ & $6(26)$ \\
\hline & Há ordenação de medicamentos conforme PEPS ( $1^{\circ}$ que entra é $1^{\circ}$ que sai)? & $18(79)$ & $5(21)$ \\
\hline
\end{tabular}

A prescrição de medicamentos, sem fundamentação em evidências e incoerente com o contexto no qual o paciente está inserido, é um potencial propul- sor de ações judiciais para obtenção de medicamentos que, muitas vezes, não agregarão valor ao tratamento do paciente e, tão-somente, onerarão o Estado(11). 
Em Caicó, a programação era anual e elaborada segundo o método do consumo histórico, baseado exclusivamente em dados de consumo, o que pode gerar equívocos, considerando que dados de consumo nem sempre são fidedignos e períodos prolongados de desabastecimento comprometem este método.

Quanto à aquisição, era realizada pelo Setor de Compras, vinculado à Secretaria Municipal de Saúde, mais especificamente, pela Comissão de Licitação, responsável pelas licitações para aquisição de medicamentos e correlatos, as quais são anuais, sendo a modalidade pregão a mais usada. Utilizavam registro técnico dos fornecedores, no entanto, não existia um catálogo com especificações técnicas dos medicamentos.

Nesse estudo, foi constatada, no momento da coleta de dados (17\%), a presença de medicamentos com prazo de validade expirado nas prateleiras das farmácias das UBS, fato também constatado em um estudo envolvendo 23 municípios de abrangência da $15^{\mathrm{a}}$ Regional de Saúde do Paraná, no qual 11 tinham medicamentos com prazo de validade expirado nas prateleiras das farmácias (12); e em Macaíba, RN, no qual todas as 22 UBS apresentavam este problema(13).

A CAF municipal era responsável pela destinação de medicamentos vencidos e avariados, via contrato estabelecido com empresa incineradora. No entanto, não existia plano de recolhimento de medicamentos vencidos e avariados formalmente descrito, o que pode contribuir para o comprometimento do controle de estoque.

Visando contribuir para ampliação de acesso e promoção do uso racional de medicamentos, em 2009 o Ministério da Saúde apresentou o software Hórus, disponibilizando-o gratuitamente a Estados e Municípios. A Portaria $n^{\circ}$ 271/13 estabelece que todos os municípios devem informatizar suas farmácias, a fim de transmitir a movimentação de medicamentos da assistência farmacêutica básica, seja via Hórus, seja via sistema Web Service (14).

Em Caicó, apenas a CAF dispunha do Hórus. As 23 UBS não foram contempladas por não possuírem estrutura mínima para a sua implantação, no caso, computador, impressora, internet e farmacêutico responsável. É imprescindível a existência de protocolos de fluxo de pedidos de medicamentos (15). Desta forma, a programação inadequada das solicitações de medicamentos das UBS poderia afetar o abastecimento da CAF e refletir diretamente no acesso do usuário ao medicamento.. Constatou-se considerável fragilidade na padronização destes fluxos com ausência na padronização dos pedidos mensais nas UBS do município.

Além disso, não o registro diário não era realizado, restringindo-se à contagem mensal das unidades dispensadas, para fundamentação de pedidos à $\mathrm{CAF}$, o que configura gerenciamento ineficaz de estoque. Um estudo realizado em 10 municípios dos Estados ES, GO, PA, RS e SE, abrangendo 30 UBS, mostrou que apenas $32 \%$ tinham registro de estoque, enfatizando que o controle de estoque é crítico na gestão da assistência farmacêutica (8).

A ausência de registro de estoque nas farmácias das UBS em questão (100\%), somada à entrega de medicamentos apenas via cartão padrão $(65 \%)$ e até mesmo sem a apresentação de prescrição (52\%) (Tabela 2) sugere que os dados de consumo não eram confiáveis.

Isso pode ser decorrente, em parte, do fato da distribuição de medicamentos nas UBS ser realizada por profissionais que não o farmacêutico (Tabela 2), portanto, não qualificados tecnicamente para a dispensação. Além disso, os funcionários não receberam treinamento $(100 \%)$, não foram encontrados nas farmácias documentos com instruções acerca da sistemática adequada de execução das atividades (100\%), os POP, e não havia recurso humano fixo nas farmácias $(100 \%)$, ou seja, o profissional disponível no momento da demanda distribuia o medicamento, o que favorecia o acesso livre à farmácia por parte de toda equipe de saúde.

Neste cenário, a coordenação de assistência farmacêutica constava em organograma da Secretaria Municipal de Saúde, mas o coordenador não era farmacêutico. Quanto ao número de farmacêuticos que atuavam na assistência farmacêutica municipal, entre 23 UBS e CAF, apenas na CAF tinha farmacêuticos lotados (dois). A carência de farmacêutico na atenção básica é relatada para vários municípios brasileiros $(13,16,17,18)$. 
A Resolução CFF no 357/01 (19) e a Portaria $n^{\circ} 3.916 / 98$ (2) apresentam a dispensação de medicamentos como atribuição indelegável do farmacêutico; e a Lei $n^{\circ}$ 13.021/14 (20) determina a obrigaatoriedade da presença do farmacêutico durante todo o horário de funcionamento nas farmácias de qualquer natureza.
Considerando o acesso como uma das dimensões da qualidade do serviço de saúde, reorientá-lo para CAF ou outra UBS que não a da área de abrangência do usuário, na ausência de determinado medicamento, compromete a qualidade do serviço de saúde quanto a esta dimensão (Tabela 2).

Tabela 2. Acesso a medicamentos nas farmácias públicas do município de Caicó, RN, Brasil.

\begin{tabular}{|l|c|}
\hline \multicolumn{1}{|c|}{ DIMENSÃO } & $\mathbf{n} / \%$ \\
\hline Documento base para entrega do medicamento & $23(100)$ \\
\hline Prescrição & $16(65)$ \\
\hline Cartão padrão & $12(52)$ \\
\hline Sem prescrição & $23(100)$ \\
\hline Responsável pela distribuição do medicamento & $17(74)$ \\
\hline Técnico em enfermagem & $8(36)$ \\
\hline Enfermeiro & \\
\hline Agente comunitário de saúde & $23(100)$ \\
\hline Equipamento de saúde para acesso a medicamento & $16(65)$ \\
\hline Outra UBS & $14(60)$ \\
\hline Farmácia privada & \\
\hline CAF & \\
\hline
\end{tabular}

Outrossim, o acesso de medicamentos, via farmácia privada (65\%) sugeriu, por um lado, uma prescrição de medicamentos em não conformidade com a REMUME, reforçada pelo fato de disponibilidade de REMUME aos prescritores somente em $48 \%$ das UBS, e por outro, a transferência de responsabilidade quanto aos medicamentos disponibilizados via Programa Aqui Tem Farmácia Popular.

Quanto à estrutura preconizada para farmácias de UBS, o mobiliário compreende mesas, cadeiras, armários fechados, ar condicionado, computador e impressora com acesso à internet, termômetro digital, lixeira com tampa e pedal, bancadas revestidas, estantes de aço, caixas organizadoras e pallets/estrados (21). Nenhuma das 23 farmácias avaliadas estava estruturada conforme preconizado. Apenas $65 \%$ possuiam ar condicionado, porém, em nenhuma foi constatado o registro de temperatura e umidade da área física, pela inexistência de termohigrômetro. Pode ser destacada, também a falta de computador e acesso à internet em $100 \%$ dos estabelecimentos.

As fragilidades constatadas também foram observadas em Macaíba, RN e Belo Horizonte, MG, destacando a infraestrutura inadequada, o compro- metimento de ventilação e controle de temperatura e umidade $(13,22)$.

\section{CONCLUSÃO}

Diante do exposto, que a assistência farmacêutica no município de Caicó era incipiente e, como tal, passível de fragilidades, o que exige maiores investimentos em recursos humanos, econômicos e infraestrutura.

A ausência do farmacêutico nas UBS é aspecto decisivo para comprometimento das etapas do ciclo da assistência farmacêutica, mas não se pode deixar de destacar a responsabilidade dos gestores municipais quanto a suas atribuições no contexto da assistência farmacêutica; no caso, viabilizar ocupação da coordenação da assistência farmacêutica por farmacêutico, além de melhorar as condições da estrutura física e operacional. Neste último caso, estruturação de sistema informatizado para controle de estoque, por exemplo.

Sendo assim, os resultados deste estudo contribuem como subsídio ao planejamento de intervenções mais resolutivas, como as supracitadas, para as fragilidades constatadas. 
1 BRASIL. Ministério da Saúde. Resolução n 338, 6 de maio de 2004. Aprova a Política Nacional de Assistência Farmacêutica. Diário Oficial da União, 20 de maio de 2004. Seção 1. p. 52.

2 BRASIL. Lei $n^{\circ}$. 8080/90, de 19 de setembro de 1990. Dispõe sobre as condições para a promoção, proteção e recuperação da saúde, a organização e o funcionamento dos serviços correspondentes e dá outras providências. Diário Oficial da União, 20 de setembro de 1990. Seção 1. p. 18055 .

3 BRASIL. Ministério da Saúde. Portaria no 3.916, de 30 de outubro de 1998. Aprova a Política Nacional de Medicamentos. Diário Oficial da União, 10 de novembro de 1998. Seção 1. p. 18.

4 BRASIL. Ministério da Saúde. Portaria no 2.436, de 21 de setembro de 2017. Aprova a Política Nacional de Atenção Básica, estabelecendo a revisão de diretrizes para a organização da Atenção Básica, no âmbito do Sistema Único de Saúde (SUS). Diário Oficial da União, $\mathrm{n}^{\circ}$ 183, 22 de setembro de 2017. Seção 1. p. 68-69.

5 Souza GS et al. Caracterização da institucionalização da assistência farmacêutica na atenção básica no Brasil. Rev Saude Publica. 2017; 51(Suppl2):1-12. DOI: 10.11606/ S1518-8787.2017051007135.

6 Farias MR, Diehl EE, Buendgens FB, Bernd KCP, Storb $\mathrm{H}$, orgs. Assistência farmacêutica no Brasil: política, gestão e clínica. v. 3. Florianópolis: Ed. da UFSC. 2016.

7 IBGE. Instituto Brasileiro de Geografia e Estatística. Cidades. 2017. [citado em 4 de fevereiro de 2018]. Disponível em: https://cidades.ibge.gov.br/brasil/rn/caico/ panorama.

8 OPAS. Organização Panamericana da Saúde. Avaliação da Assistência Farmacêutica no Brasil. Brasília: Ministério da Saúde, 2005.

9 Donabedian A. La Calidad de la Atención Médica: definiciones - métodos de evaluación. Ediciones Científicas. La prensa Mexicana, S. A, 1984.

10 Lima MG, Álvares J, Guerra Junior AA, Costa EA, Guibu IA, Soeiro OM, Leite SN, Karnikowski MGO, Costa KS, Acurcio FA. Indicadores relacionados ao uso racional de medicamentos e seus fatores associados. Rev Saude Pub. 2017;51(Suppl2):1-9. DOI: 10.11606/s15188787.2017051007137.

11 Santi LQ. Prescrição: o que levar em conta? Uso Racional de Medicamentos: fundamentação em condutas terapêuticas e nos macroprocessos da Assistência Farmacêutica. Série Uso Racional de Medicamentos. Organização Pan-Americana de Saúde, 2016; 1(14):1-11.
12 Volpato DC, Padial RB. Avaliação da assistência farmacêutica em municípios de uma regional de saúde do Paraná. Rev Saúde e Pesq. 2014;7(2):221-232.

13 Simão RWTS, Batista AA. Armazenamento de medicamentos em unidades básicas de saúde de Macaíba, RN, Brasil. Infarma - Ciências Farmac. 2016;28(1):40-46. DOI: 10.14450/2318-9312.v28.e1.a2016.pp40-46.

14 BRASIL. Portaria n ${ }^{\circ}$ 271, de 27 de fevereiro de 2013. Institui a Base Nacional de Dados de ações e serviços da Assistência Farmacêutica e regulamenta o conjunto de dados, fluxo e cronograma de envio referente ao Componente Básico da Assistência Farmacêutica no âmbito do Sistema Único de Saúde (SUS). 2013. [citado em 11 de novembro de 2018]. Disponível em: http://bvsms.saude.gov. br/bvs/saudelegis/gm/2013/prt1555_30_07_2013.html.

15 Silva TFK. Distribuição de medicamentos da central de abastecimento farmacêutico para as unidades de saúde: com ênfase nas falhas do processo. Facider Rev Científica. $2015 ;(8): 1-28$.

16 Margarino-Torres R, Pepe VLE, Oliveira MA, Osorio-de-Castro CGS. Medicamentos essenciais e processo de seleção em práticas de gestão da assistência wfarmacêutica em estados e municípios brasileiros. Ciênc. Saúde Coletiva. 2014; 19(9):3859-3868. DOI: 10.1590/141381232014199.12162013.

17 Paiva CR, Batista AM. Distribuição de medicamentos em serviço de saúde de um município do Rio Grande do Norte, Brasil. J Assist Farmac Farmacoecon. 2017;2(3):1222. DOI: 10.22563/2525-7323.2017.v2.n3.p12-22.

18 VieiraFS,ZucchiP.Gestão daassistência farmacêutica:análisedasituaçãodealgunsmunicípios. Tempus, actasdesaúde colet. 2014;8(4):11-29. DOI: 10.18569/tempus.v8i4.1581.

19 CFF. Conselho Federal de Farmácia. Resolução n 357, de 20 de abril de 2001. Aprova o regulamento técnico das Boas Práticas de Farmácia. [citado em 17 de dezembro de 2017]. Disponível em: http://www.cff.org.br.

20 BRASIL. Lei $n^{\circ} 13021$, de 8 de agosto de 2014. Dispõe sobre o exercício e a fiscalização das atividades farmacêuticas. Diário Oficial da União, 11 de agosto de 2014. Seção 1. p. 1.

21 BRASIL. Ministério da Saúde. Diretrizes para estruturação de farmácias no âmbito do Sistema Único de Saúde. Brasília: Ministério da Saúde, 2009.

22 Dutra KR, Martins UCM, Lima MG. Condições de armazenamento de medicamentos nas unidades básicas de saúde do distrito sanitário nordeste de Belo Horizonte, Brasil. Infarma - Ciências Farm. 2018; 30(2):130-133. DOI: 10.14450/2318-9312.v30.e2.a2018.pp130-133 